INFLAMMATORY BOWEL DISEASE

\title{
Increased risk of lymphoma among inflammatory bowel disease patients treated with azathioprine and 6-mercapto- purine
}

\author{
A Kandiel, A G Fraser, B I Korelitz, C Brensinger, J D Lewis
}

Gut 2005;54:1121-1125. doi: 10.1136/gut.2004.049460

See end of article for authors' affiliations

.....................

Correspondence to: Dr J D Lewis, University of Pennsylvania, 720 Blockley Hall, 423 Guardian Drive, Philadelphia, PA 191046021, USA; jlewis@ cceb.med.upenn.edu

Revised version received 22 December 2004 Accepted for publication 4 January 2005
Background: Inflammatory bowel disease (IBD) is commonly treated with immunomodulators such as azathioprine and 6-mercaptopurine (6-MP). Studies examining lymphoma risk in IBD patients treated with these medications have been underpowered and have yielded conflicting conclusions.

Aims: The purpose of this meta-analysis was to provide a more precise estimate of the relative risk of lymphoma among IBD patients treated with azathioprine or 6-MP.

Methods: Studies were included if they were English language, full article, cohort studies specifically designed to evaluate cancer as an adverse outcome of treatment with azathioprine or 6-MP. Pooled standardised incidence ratios were calculated to estimate the relative risk of lymphoma associated with therapy. Heterogeneity was assessed using Poisson regression. Sensitivity analyses examined the influence of individual studies on risk estimate and heterogeneity statistics.

Results: Six studies were identified that met our inclusion criteria. When the data were combined across all studies, the pooled relative risk was 4.18 (95\% confidence interval $2.07-7.51 ; 11$ observed cases, 2.63 expected). Sensitivity analysis showed that exclusion of any one study had a relatively small effect on the pooled relative risk estimate (range 3.49-5.21) but excluding either the study with the highest or lowest estimated relative risk eliminated the statistically significant heterogeneity.

Conclusions: Our data suggest an approximate fourfold increased risk of lymphoma in IBD patients treated with azathioprine/6-MP. The increased risk of lymphoma could be a result of the medications, the severity of the underlying disease, or a combination of the two. a nflammatory bowel disease (IBD) is treated with multiple agents, including steroids, 5-ASA agents, biological agents, and immunomodulators such as azathioprine and 6-mercaptopurine (6-MP). IBD, in and of itself, does not appear to be associated with an increased risk of lymphoma in most population based studies. ${ }^{1-7}$ However, immunosuppression, either disease mediated as in AIDS, or iatrogenic as in the post-transplant state, is associated with an increased risk of lymphoma. ${ }^{8-10}$ Furthermore, a few studies have suggested a small increased risk of lymphoma among patients with rheumatoid arthritis treated with azathioprine or 6-MP. ${ }^{11-13}$ Several studies have examined the risk of lymphoma in IBD patients treated with immunomodulators, particularly azathioprine and 6-MP. ${ }^{12}{ }^{14-17}$ The authors of these studies have come to conflicting conclusions regarding the risk of lymphoma among IBD patients treated with immunomodulator therapy. Importantly, each of these studies was underpowered to identify a small increased risk of lymphoma with therapy. The technique of meta-analysis can be used to combine the results of similar studies to increase statistical power and increase the precision of relative risk estimates. The aim of this meta-analysis was to determine whether there is an increased risk of lymphoma in IBD patients treated with azathioprine or 6-MP.

\section{MATERIALS AND METHODS}

\section{Inclusion criteria}

For a study to be included in this meta-analysis it had to meet the following inclusion criteria: the study must be a cohort study, the exposed group must have received azathioprine or 6-MP, the study must have been specifically designed to evaluate cancer as an adverse outcome of treatment, and the study must have been a full article published in the English language. Studies that did not meet all of these criteria were excluded.

\section{Search strategies}

Multiple search strategies were used in Medline to find articles that would meet the above inclusion criteria. The searches were limited to articles published in the English language. The initial search used the following keywords: (azathioprine or 6-MP or 6-mercaptopurine or immunosuppress\$) and (Crohn's disease or ulcerative colitis or inflammatory bowel disease) and (lymphoma or cancer or malignancy). Keywords were used in preference to MeSH terms in order to increase the sensitivity of our search strategy. This search yielded 80 articles, of which 76 were excluded by title and/or abstract and/or review of the full article, and four were retained. ${ }^{1}{ }^{14}{ }^{15} 17$ An additional search using just the keywords azathioprine and lymphoma yielded an additional article that met the inclusion criteria. ${ }^{12}$ Other search strategies did not yield any additional relevant articles. A comprehensive review of article reference lists, however, did result in an additional article for analysis. ${ }^{16}$ All articles were reviewed by two investigators (AK and JL). Discrepancies were resolved by discussion and re-review of the articles. Data were abstracted on year of publication, journal, type of study (single centre $v$ population based), number of patients with IBD, number of patients with Crohn's disease, number of patients with ulcerative colitis, mean/median duration of therapy with azathioprine and/or

Abbreviations: IBD, inflammatory bowel disease; 6-MP, 6-mercaptopurine; SIR, standardised incidence ratio; HD, Hodgkin's disease; NNH, number needed to harm one additional patient per year; SEER,

Surveillance Epidemiology and End Results 
Table 1 Summary of studies included in the meta-analysis

\begin{tabular}{|c|c|c|c|c|c|c|}
\hline & Connell $^{14}$ & Farrell $^{15}$ & Fraser $^{16}$ & Kinlen $^{12}$ & Korelitz $^{17}$ & Lewis' \\
\hline Year & 1994 & 2000 & 2002 & 1985 & 1999 & 2001 \\
\hline Setting & Single centre & Single centre & Single centre & Single centre & Single centre & Population based \\
\hline Total No of IBD patients & 755 & $238^{*}$ & 626 & 321 & 486 & 1465 \\
\hline \% with CD & 60 & 46 & 43 & $\mathrm{~N} / \mathrm{R}$ & 67 & 57 \\
\hline \% with UC & 40 & 54 & 57 & $\mathrm{~N} / \mathrm{R}$ & 33 & 43 \\
\hline Mean/median duration of treatment & $12.5 \mathrm{mo}$ & 1.82 y & $2.26 y$ & $N / R$ & $4.4 y$ & $2 y$ \\
\hline Medication studied & AZA & AZA & AZA & AZA & 6-MP & AZA \\
\hline Mean/median dose or range & $2 \mathrm{mg} / \mathrm{kg} / \mathrm{day}$ & $2-2.5 \mathrm{mg} / \mathrm{kg} /$ day & $1.65 \mathrm{mg} / \mathrm{kg} /$ day & $N / R$ & $12.5-100 \mathrm{~g} /$ day & 106 mg/day \\
\hline Mean/median duration of follow up & $9 y$ & $6.9 y$ & 6.9 y & $N / R$ & 5.9 y & 2.87 y \\
\hline Outcome & NHL & $\mathrm{NHL}$ & NHL and HD & $\mathrm{NHL}$ & NHL and HD & $\mathrm{NHL}$ and $\mathrm{HD}$ \\
\hline Expected No of lymphomas & 0.52 & 0.05 & 0.65 & 0.16 & 0.61 & 0.64 \\
\hline Observed No of lymphomas & 0 & $2 \dagger$ & 3 & 2 & 3 & 1 \\
\hline SIR $\ddagger(95 \% \mathrm{CI})$ & $0(-)$ & $37.5(3.5-137.7)$ & $4.6(0.9-13.7)$ & $12.5(1.2-46.0)$ & $4.9(0.9-14.5)$ & $1.6(0.0006-9.0)$ \\
\hline
\end{tabular}

IBD, inflammatory bowel disease; UC, ulcerative colitis; CD, Crohn's disease; NHL, non-Hodgkin lymphoma; HD, Hodgkin's disease; AZA, azathioprine; 6-MP, 6-mercaptopurine; $95 \% \mathrm{Cl}, 95 \%$ confidence interval.

*Treated with immunomodulators.

†Two additional lymphomas observed in patients treated with methotrexate and ciclosporin.

$N / R$, not reported.

$\ddagger S I R$, standardised incidence ratio for lymphoma. Because of rounding of the expected number of lymphomas, SIR does not exactly equal observed number of lymphomas divided by expected number of lymphomas.

6-MP, mean/median dose, mean median duration of follow up, expected number of patients with lymphoma, observed number of patients with lymphoma, and type of lymphoma.

\section{Statistical analyses}

In order calculate a pooled estimate of the relative risk of lymphoma, it was necessary to have all studies analysed in the same manner. All but two studies ${ }^{16}{ }^{17}$ calculated standardised incidence ratios (SIRs) using indirect standardisation. ${ }^{18}$ In this method observed numbers of cancers in each cohort are compared with the expected number of cases of lymphoma based on age and sex specific rates for the general population that gave rise to the study cohort; $95 \%$ confidence intervals (CI) are calculated assuming that the incidence of lymphoma follows a Poisson distribution. If the 95\% CI excludes 1.0, this is consistent with a statistically significant increase or decrease in the risk of lymphoma with a type 1 error of less than 0.05 .

For studies by Fraser and colleagues ${ }^{16}$ and Korelitz and colleagues, ${ }^{17}$ we reanalysed the primary data to calculate the SIRs. For the study by Fraser and colleagues, ${ }^{16}$ we used the primary data from the original study and compared these data with lymphoma rates for England and Wales. ${ }^{19}$ Thus the SIR was calculated as the ratio of observed lymphomas divided by the expected number of lymphomas based on the age and sex distribution of the study population. Confidence intervals were estimated assuming that the incidence of lymphoma follows a Poisson distribution.

For the study by Korelitz and colleagues, ${ }^{17}$ the data set that was used in the original publication was no longer available. As a result, we used more up to date data obtained from the study team. In analysing the data from Korelitz et al, duration of follow up for each patient was calculated from the date of first azathioprine/6-MP prescription to the latter of the date of the last azathioprine/6-MP use or the date of the last endoscopic procedure. For patients who developed lymphoma, the follow up period was censored at the date of lymphoma. The observed number of lymphomas was compared with the expected number, which was derived from rates reported by the Surveillance Epidemiology and End Results (SEER) cancer registry. ${ }^{20}$ Confidence intervals were again estimated assuming that the incidence of lymphoma follows a Poisson distribution.

The study by Farrell and colleagues ${ }^{15}$ included two cases of lymphoma in patients treated exclusively with methotrexate and/or ciclosporin. For this study, we included only those lymphoma patients treated with azathioprine or 6-MP, and excluded patients treated exclusively with methotrexate and/ or ciclosporin. We reduced the expected number of lymphomas in proportion to the fraction of the total cohort of patients treated with immunomodulators other than azathioprine or 6-MP (expected 0.06 in original article, reduced to 0.053 in our analysis).

In the study by Kinlen, ${ }^{12}$ data were not presented on the subgroup of patients with IBD. However, data were available on all patients without rheumatoid arthritis. Therefore, in our analyses we used the data excluding all rheumatoid arthritis patients. As such, we included data on a total of 991 patients of whom 321 (32\%) had IBD.

Pooled SIRs and 95\% CI were estimated by summing the observed and expected numbers of lymphomas across studies. To examine the studies for evidence of heterogeneity, we used the deviance statistic derived from Poisson regression models, including each of the studies. A p value of less than 0.05 was considered evidence of statistically significant heterogeneity. To determine which studies contributed to the observed heterogeneity and to estimate the impact of these studies on the pooled SIR estimate, we performed a series of sensitivity analyses in which the pooled SIR and the deviance statistic were recalculated after excluding each study.

Secondary analyses were performed examining the relative risk of non-Hodgkin lymphoma. For these analyses, we recalculated the SIRs for the Lewis, Fraser, and Korelitz studies using only the expected rates of non-Hodgkin lymphoma. Patients who developed Hodgkin's disease were censored at the time of lymphoma diagnosis.

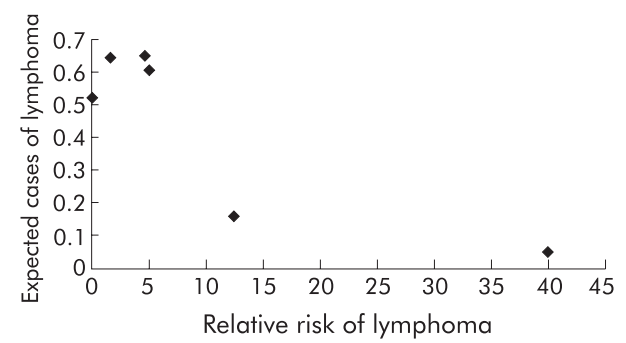

Figure 1 Heterogeneity among the studies. Relative risk of lymphoma against the expected number of cases of lymphoma in each study. The expected number of cases was strongly correlated with total follow up time for the cohort. There was a lower estimated relative risk among large studies and higher estimates among smaller studies. 
Table 2 Sensitivity analysis of lymphoma risk when individual studies were excluded from the analysis

\begin{tabular}{lll}
\hline $\begin{array}{l}\text { Study } \\
\text { excluded }\end{array}$ & SIR $(95 \% \mathrm{Cl})$ & $\begin{array}{l}\text { Test of heterogeneity } \\
\text { p value* }\end{array}$ \\
\hline Connell $^{14}$ & $5.21(2.59-9.35)$ & 0.09 \\
Kinlen $^{12}$ & $3.64(1.65-6.94)$ & 0.03 \\
Farrell $^{15}$ & $3.49(1.58-6.66)$ & 0.12 \\
Lewis $^{1}$ & $5.02(2.39-9.27)$ & 0.03 \\
Fraser $^{16}$ & $4.03(1.72-7.98)$ & 0.01 \\
Korelitz $^{17}$ & $3.95(1.69-7.84)$ & 0.01 \\
\hline
\end{tabular}

SIR, standardised incidence ratio (expected/observed) ${ }^{*} \mathrm{p}<0.05$ implies there is significant heterogeneity among the studies included in the analysis.

Three studies provided sufficient data to compare lymphoma risk in azathioprine or 6-MP treated IBD patients to that in IBD patients not treated with these medications. ${ }^{1}{ }^{15} 16$ The results of these studies were pooled using MantelHaenszel methods with weights proportional to the inverse variance, as implemented in the STATA "ir" routine (STATA version 8.2, College Station, Texas, USA). Two of the studies $^{116}$ allowed for calculation of age adjusted relative risk estimates. Results of these studies were pooled using the random effects summary estimate of the data, as implemented in the STATA "meta" routine (STATA version 8.2).

\section{Ethics approval}

This study was approved by the Institutional Review Board of the University of Pennsylvania.

\section{RESULTS}

Six studies met our inclusion criteria (table 1).

\section{Relative risk of lymphoma}

When the data were combined across all of the included studies, the total number of observed cases of lymphoma was 11 , and the total number of expected cases was 2.63, resulting in an SIR of 4.18 (95\% CI 2.07-7.51). However, as can be seen in fig 1 , there was significant heterogeneity among the studies (deviance statistic $\mathrm{p}=0.03$ ).

Sensitivity analyses demonstrated that the significant heterogeneity in pooled analyses was explained by the extreme difference in results of the studies of Connell and colleagues $^{14}$ and Farrell and colleagues. ${ }^{15}$ When either of these studies was excluded from the analysis, the test for heterogeneity was no longer statistically significant (table 2 ). Importantly, in these analyses, exclusion of any one study had a relatively small effect on the pooled relative risk estimate (range 3.49-5.21). Furthermore, excluding any one study did not eliminate the statistical significance of the pooled estimate of the relative risk of lymphoma.

Because the study of Kinlen ${ }^{12}$ included patients without IBD, we performed additional sensitivity analyses excluding this study and the two previously identified outliers. When both the Kinlen study ${ }^{12}$ and the Connell and colleagues study ${ }^{14}$ were excluded from the analysis, the SIR was 4.61 (95\% CI 2.09-8.79); when the Kinlen study ${ }^{12}$ and the Farrell and colleagues study ${ }^{15}$ were excluded from the analysis, the SIR was 2.90 (95\% CI 1.15-6.00).

Three studies provided sufficient data to directly compare azathioprine or 6-MP treated IBD patients with IBD patients who had not received this therapy. ${ }^{15} 16$ The pooled analysis yielded a combined relative risk of 2.92 (95\% CI 1.05-8.13, test for heterogeneity $\mathrm{p}=0.18$ ). If we excluded the Farrell study, ${ }^{15}$ the combined relative risk estimate was 2.03 (95\% CI 0.66-6.29). Using age adjusted estimates of the relative risk from the studies of Fraser and colleagues ${ }^{16}$ and Lewis and colleagues, ${ }^{1}$ the pooled analysis yielded a slightly higher relative risk estimate of 3.11 (95\% CI 0.66-14.62).

To help place the significance of our findings into a clinical context, we estimated the number of person years of follow up after therapy with azathioprine or 6-MP needed to result in one additional case of lymphoma. Based on the results from our meta-analysis, we performed this calculation for a range of relative risk estimates (low of 2.0 to high of 6.0) (table 3). Based on these calculations, assuming a relative risk of lymphoma of 4.0, the number of patients needed to be treated to cause one additional lymphoma per year ranged from approximately 4357 persons aged 20-29 years to 355 persons aged 70-79 years.

In secondary analyses, we focused specifically on the relative risk of non-Hodgkin lymphoma. There was significant heterogeneity $(p=0.01)$ among the studies when we examined only the risk of non-Hodgkin lymphoma, with two studies estimating very high relative risks of lymphoma (Farrell and colleagues ${ }^{15}$ SIR 37.4 and Kinlen ${ }^{12}$ SIR 12.5) while two studies (Lewis and colleagues ${ }^{1}$ and Connell and colleagues ${ }^{14}$ ) observed no cases of non-Hodgkin lymphoma, thus resulting in SIR estimates of zero. Combining all studies, there were nine cases of non-Hodgkin lymphoma compared with an expected 2.30 (SIR 3.92; 95\% CI 1.78-7.47). The increased relative risk of lymphoma and the test for heterogeneity remained statistically significant when any one study was excluded. The lowest estimate was observed when the study by Farrell and colleagues ${ }^{15}$ was excluded (SIR 3.12; 95\% CI 1.24-6.46). Excluding the study by Lewis and colleagues $^{1}$ resulted in the highest SIR (SIR 5.07; 95\% CI 2.30-9.66). When we dropped the studies by both Kinlen ${ }^{12}$ and Farrell and colleagues, ${ }^{15}$ the resulting estimated SIR was 2.40 (95\% CI 0.76-5.64). Because of the heterogeneity, these secondary pooled analyses need to be viewed with caution.

\section{Case descriptions}

Overall, there were 11 cases of lymphoma, of which two were Hodgkins (table 4). Patients had received a median of 14 months of therapy prior to the diagnosis of lymphoma (range 6-94 months). Of the nine non-Hodgkins lymphomas, four originated in the bowel, one originated in the central nervous system, two originated elsewhere, and the site was not reported for the remaining two lymphomas.

Table 3 Number needed to treat to cause one additional lymphoma per year

\begin{tabular}{lllll}
\hline Age $(\mathbf{y})$ & $\begin{array}{l}\text { Lymphoma } \\
\text { incidence* }\end{array}$ & $\begin{array}{l}\text { NNH if relative risk } \\
\text { of lymphoma = 2 }\end{array}$ & $\begin{array}{l}\text { NNH if relative risk } \\
\text { of lymphoma = 4 }\end{array}$ & $\begin{array}{l}\text { NNH if relative risk } \\
\text { of lymphoma =6 }\end{array}$ \\
\hline $20-29$ & 7.65 & 13072 & 4357 & 2614 \\
$30-39$ & 10.70 & 9346 & 3115 & 1869 \\
$40-49$ & 16.60 & 6024 & 2008 & 1205 \\
$50-59$ & 29.60 & 3378 & 1126 & 676 \\
$60-69$ & 56.45 & 1771 & 591 & 354 \\
$70-79$ & 93.90 & 1065 & 355 & 213 \\
\hline
\end{tabular}

*Incidence rates per 100000 person years from 1996-2000 (SEER data ${ }^{17}$ )

$\mathrm{NNH}$, number needed to harm one additional patient per year. 
Table 4 Case summaries of lymphoma patients

\begin{tabular}{lllllll}
\hline $\begin{array}{l}\text { Author/ } \\
\text { patient No }\end{array}$ & $\begin{array}{l}\text { Age } \\
\text { (y) }\end{array}$ & Sex & CD v UC & $\begin{array}{l}\text { Duration of } \\
\text { AZA/6-MP therapy } \\
\text { (months) }\end{array}$ & NHL/HD & $\begin{array}{l}\text { Location of } \\
\text { NHL }\end{array}$ \\
\hline Farrell $^{15} \# 1$ & 53 & $\mathrm{M}$ & $\mathrm{UC}$ & 14 & $\mathrm{NHL}$ & Bowel \\
Farrell $^{15} \# 2$ & 34 & $\mathrm{~F}$ & $\mathrm{CD}$ & 9 & $\mathrm{NHL}$ & Bowel \\
Fraser $^{16} \# 1$ & 43 & $\mathrm{M}$ & $\mathrm{UC}$ & 72 & $\mathrm{NHL}$ & Bowel \\
Fraser $^{16} \# 2$ & 19 & $\mathrm{M}$ & $\mathrm{UC}$ & 4 & $\mathrm{HD}$ & - \\
Fraser $^{16} \# 3$ & 52 & $\mathrm{~F}$ & $\mathrm{UC}$ & 94 & $\mathrm{NHL}$ & Other \\
Kinlen $^{12} \# 1-2$ & $\mathrm{~N} / \mathrm{R}$ & $\mathrm{N} / \mathrm{R}$ & $\mathrm{N} / \mathrm{R}$ & $\mathrm{N} / \mathrm{R}$ & $\mathrm{N} / \mathrm{R}$ & $\mathrm{N} / \mathrm{R}$ \\
Korelitz $^{17} \# 1$ & 69 & $\mathrm{M}$ & $\mathrm{CD}$ & 40 & $\mathrm{NHL}$ & Bowel \\
Korelitz $^{17} \# 2$ & 53 & $\mathrm{M}$ & $\mathrm{CD}$ & 6 & $\mathrm{CNS}$ \\
Korelitz $^{17} \# 3$ & 50 & $\mathrm{M}$ & $\mathrm{CD}$ & 87 & $\mathrm{NHL}$ & Other \\
Lewis $^{17} \# 1$ & 47 & $\mathrm{M}$ & $\mathrm{UC}$ & 10 & $\mathrm{HD}$ & - \\
\hline \multicolumn{7}{c}{ CD, Crohn's disease; UC, ulcerative colitis; NHL, non-Hodgkin lymphoma; HD, Hodgkin's disease; AZA, } \\
azathioprine; 6-MP, 6-mercaptopurine; N/R, not reported. & &
\end{tabular}

\section{DISCUSSION}

Most previous research suggests that the risk of lymphoma among patients with IBD is similar to that in the general population. ${ }^{1-7}$ In contrast, this meta-analysis demonstrates that there is an approximate fourfold increased risk of lymphoma in the subgroup of IBD patients treated with azathioprine and/or 6-MP relative to the lymphoma rate expected in the general population. These findings are consistent with prior research demonstrating a modest increased risk of lymphoma among patients receiving these medications for rheumatoid arthritis. ${ }^{11-13}$ The increased risk of lymphoma among IBD patients is markedly lower than that observed after organ transplantation, ${ }^{9}{ }^{10}$ a condition in which much greater levels of immunosuppression are achieved.

Another way to place these results into a clinical context is to ask how great of a risk of lymphoma is necessary for the risks of therapy to outweigh the benefits. Lewis et al previously conducted a decision analysis using a Markov model to assess the efficacy of alternative management strategies for maintaining remission in patients with Crohn's disease. ${ }^{21}$ That study showed that azathioprine must result in a greater than 9.8-fold increased risk of lymphoma for therapy with alternative medications to be the preferred treatment strategy, assuming that Crohn's disease does not itself lead to a baseline increased risk of lymphoma. ${ }^{21}$ Using this model, we can then assume that a fourfold increased risk of lymphoma does not preclude the use of azathioprine and/ or 6-MP in the treatment of IBD, especially in young patients.

An important objective of meta-analysis is to look for evidence of heterogeneity among studies and to determine whether differences in study design explain the heterogeneity. We observed significant heterogeneity among studies in both our primary and secondary analyses. The greatest difference was between the results of studies by Connell and colleagues ${ }^{14}$ and Farrell and colleagues. ${ }^{15}$ Both studies were set in single centres and had similar durations of therapy, durations of follow up, and dose. The most obvious difference was sample size. The study by Farrell and colleagues $^{15}$ was the smallest and had the lowest expected number of lymphomas. Because the expected number of lymphomas was approximately 0.05 , every case of lymphoma increased the relative risk estimate by approximately 20 . Because of the small sample size, the study by Farrell and colleagues $^{15}$ produced a less precise estimate of the relative risk than the other studies. Thus while we agree with Farrell's conclusions that IBD patients treated with azathioprine/6-MP appear to be at increased risk of lymphoma, the magnitude of that increased risk appears to be far lower than that estimated by Farrell and colleagues. ${ }^{15}$

As with all meta-analyses, we designed specific inclusion and exclusion criteria prior to initiating our study. Our decision to focus exclusively on studies that included cancer as an outcome of interest was implemented to avoid misclassification bias. Studies designed to look at other outcomes may examine cancer as a secondary outcome, or more commonly as an adverse event. Data collection for these secondary outcomes or adverse events may be less complete. If so, inclusion of such studies may bias the results toward the null hypothesis.

It is possible that relevant studies could have been missed during the literature searches. Although we used multiple different combinations in our Medline search to increase our sensitivity and carefully reviewed reference lists from all studies, we limited our search to English language studies.

Meta-analyses may be biased by inability to identify unpublished studies. Our data support the possibility that publication bias may have existed. We observed lower relative risk estimates among large studies and higher estimates among smaller studies. This suggests that some smaller studies showing an increased risk of lymphoma may have been published while small studies demonstrating no increased risk remain unpublished. Importantly, small studies contribute relatively little weight in the analyses. For example, in our sensitivity analysis, where we exclude the study by Farrell and colleagues, ${ }^{15}$ the estimated relative risk only decreased from 4.18 to 3.49 .

Azathioprine/6-MP tend to be used in more severe cases of IBD. It is possible that the increased risk of lymphoma with immunomodulator therapy seen in these studies is confounded by the severity of IBD (that is, those who are at the greatest risk for lymphoma are the same population that are most likely to receive immunomodulator therapy). Arguing against confounding by indication are the results of a previous cohort study addressing the risk of lymphoma among patients with IBD. In the study by Lewis and colleagues, ${ }^{1}$ IBD patients with lymphoma were no more likely than IBD patients without lymphoma to have used 5-ASA medications, steroids, or to have undergone surgery in the previous two years. Thus, in that study, severity of IBD did not appear to be strongly associated with lymphoma risk. None the less, a conservative interpretation of our data is that IBD patients who receive immunomodulator medications are at higher risk of lymphoma than the general population, and that this increased risk could be due to the medication, disease activity, or both.

Meta-analyses are limited by the quality of the published data. Several of the studies included in our analyses had potential limitations. For example, the study by Farrell and colleagues ${ }^{15}$ had a small sample size, and as such relatively imprecise relative risk estimates. Likewise, we elected to include the study by Kinlen ${ }^{12}$ although only one third of the non-rheumatoid arthritis patients analysed had IBD. However, when the Farrell and Kinlen studies were excluded from the analysis, we obtained similar results. 
All of the studies included in our meta-analysis compared the incidence of lymphoma among IBD patients treated with immunomodulator therapy to that in the general population. Justification for this approach comes from multiple population based studies that support a lack of association between IBD and lymphoma. ${ }^{1-7}$ Thus the incidence of lymphoma in the general population should approximate that observed in IBD populations. Further evidence to support this comes from our pooled analysis of the studies with a second control group composed of patients with IBD but not treated with immunomodulator therapy. ${ }^{1516}$ Our analysis combining these studies yielded pooled relative risk estimates close to our estimate from the primary analyses.

It is unclear how the risk of lymphoma changes when therapy is discontinued, and whether the risk is dose related. The studies included in our meta-analysis all continued to follow patients after azathioprine/6-MP were discontinued. If immunosuppression is the primary mechanism leading to lymphoma, it is plausible that the risk of lymphoma would return to normal after the medications are discontinued. To the extent that this is true, individual studies and our metaanalysis may have underestimated the risk of lymphoma during therapy with azathioprine/6-MP. Regardless, our estimates should remain valid for the long term follow up of patients treated with these medications.

Some patients in our studies were treated with very low doses of immunomodulators, below that often recommended as appropriate for treatment of $\mathrm{IBD}^{22}$ Since the degree of immunosuppression appears to be related to the risk of lymphoma, this would tend to bias our results toward underestimate of the relative risk of lymphoma associated with immunomodulator therapy at currently recommended doses.

In conclusion, our data suggest an approximate fourfold increased risk of lymphoma in IBD patients treated with azathioprine/6-MP. This is consistent with relative risk estimates observed in previous studies among patients with rheumatoid arthritis. Our data suggest that one additional lymphoma will occur every 300 to 4500 years after therapy with azathioprine or 6-MP, depending on the age of the patient. Because these data were obtained from observational studies, it is not possible to fully exclude the possibility that the increased risk of lymphoma is associated with the severity of the disease, rather than being caused by the medications. Regardless, given the magnitude of the association, even if the increased risk is entirely attributable to the medications, it is unlikely to outweigh the potential benefits of these medications for most patients. In fact, to the extent that the observed increased risk of lymphoma is in part or entirely attributable to the severity of the underlying IBD, the benefit to risk ratio for these medications would be even greater.

\section{ACKNOWLEDGEMENT}

This study was supported in part by a grant from the National Institutes of Health (grant K08-DK02589 to JDL). The funding agency had no input into the design, interpretation of the results, or presentation of the data.

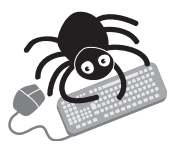

Conflict of interest: declared (the declaration can be viewed on the Gut website at http://www.gutjnl. com/supplemental)

\section{Authors' affiliations}

A Kandiel, Department of Gastroenterology and Hepatology, The Cleveland Clinic, Cleveland, Ohio, USA

A G Fraser, Department of Medicine, University of Auckland, Auckland, New Zealand

B I Korelitz, Department of Medicine, Lenox Hill Hospital, New York, USA

C Brensinger, Department of Biostatistics and Epidemiology, and Center for Clinical Epidemiology and Biostatistics, University of Pennsylvania, Philadelphia, PA

J D Lewis, Department of Medicine, Department of Biostatistics and Epidemiology, and Center for Clinical Epidemiology and Biostatistics, University of Pennsylvania, Philadelphia, PA, USA

\section{REFERENCES}

1 Lewis JD, Bilker WB, Brensinger C, et al. Inflammatory bowel disease is not associated with an increased risk of lymphoma. Gastroenterology 2001;121:1080-7.

2 Ekbom A, Helmick C, Zack M, et al. Extracolonic malignancies in inflammatory bowel disease. Cancer 1991;67:2015-19

3 Karlen $\mathbf{P}$, Lofberg $\mathrm{R}$, Brostrom $\mathrm{O}$, et al. Increased risk of cancer in ulcerative colitis: a population-based cohort study. Am J Gastroenterol 1999:94:1047-52.

4 Persson PG, Karlen P, Bernell O, et al. Crohn's disease and cancer: a population-based cohort study. Gastroenterology 1994;107:1675-9.

5 Loftus E, Tremaine W, Habermann T, et al. Risk of lymphoma in inflammatory bowel disease. Am J Gastroenterol 1998;95:2308-12.

6 Bernstein CN, Blanchard JF, Kliewer E, et al. Cancer risk in patients with inflammatory bowel disease: a population-based study. Cancer 2001;91:854-62.

7 Palli D, Trallori G, Bagnoli S, et al. Hodgkin's disease risk is increased in patients with ulcerative colitis. Gastroenterology 2000;1 19:647-53.

8 Kinlen L. Immunologic factors, including AIDS. In: Schottenfeld D, Fraumeni D, eds. Cancer epidemiology and prevention. New York: Oxford University Press, 1996:532-45.

9 Opelz G, Henderson R. Incidence of non-Hodgkin lymphoma in kidney and heart transplant recipients. Lancet 1993;342:1514-16.

10 Wilkinson AH, Smith JL, Hunsicker LG, et al. Increased frequency of posttransplant lymphomas in patients treated with cyclosporine, azathioprine, and prednisone. Transplantation 1989;47:293-6.

11 Silman AJ, Petrie J, Hazleman B, et al. Lymphoproliferative cancer and other malignancy in patients with rheumatoid arthritis treated with azathioprine: a 20 year follow up study. Ann Rheum Dis 1988;47:988-92.

12 Kinlen $\mathrm{L}$. Incidence of cancer in rheumatoid arthritis and other disorders after immunosuppressive treatment. Am J Med 1985;78:44-9.

13 Asten P, Barrett J, Symmons D. Risk of developing certain malignancies is related to duration of immunosuppressive drug exposure in patients with rheumatic diseases. J Rheumato 1999;26:1705-14

14 Connell WR, Kamm MA, Dickson M, et al. Long-term neoplasia risk after azathioprine treatment in inflammatory bowel disease. Lancet 1994:343:1249-52.

15 Farrell RJ, Ang Y, Kileen P, et al. Increased incidence of non-Hodgkin's lymphoma in inflammatory bowel disease patients on immunosuppressive therapy but overall risk is low. Gut 2000;47:514-19.

16 Fraser AG, Orchard TR, Robinson EM, et al. Long-term risk of malignancy after treatment of inflammatory bowel disease with azathioprine. Aliment Pharmacol Ther 2002;16:1225-32.

17 Korelitz BI, Mirsky FJ, Fleisher MR, et al. Malignant neoplasms subsequent to treatment of inflammatory bowel disease with 6-mercaptopurine. Am J Gastroenterol 1999;94:3248-53.

18 Breslow NDN. Statistical methods in cancer research. Lyon: International Agency for Research on Cancer, 1987.

19 Parkin D, Muir C, Whelan S, et al. Cancer incidence in five continents. Lyon: International Agency for Research on Cancer, 1992.

20 Ries LKC, Hankey B, Miller B, et al. SEER Cancer Statistics Review, 19731996. Bethesda, MD: National Cancer Institute, 1999.

21 Lewis J, Schwartz S, Lichtenstein G. Azathioprine for maintenance of remission in Crohn's disease: benefits outweigh the risk of lymphoma. Gastroenterology 2000;118:1018-24.

22 Sandborn WJ. Azathioprine: state of the art in inflammatory bowel disease. Scand J Gastroenterol Suppl 1998;225:92-9. 